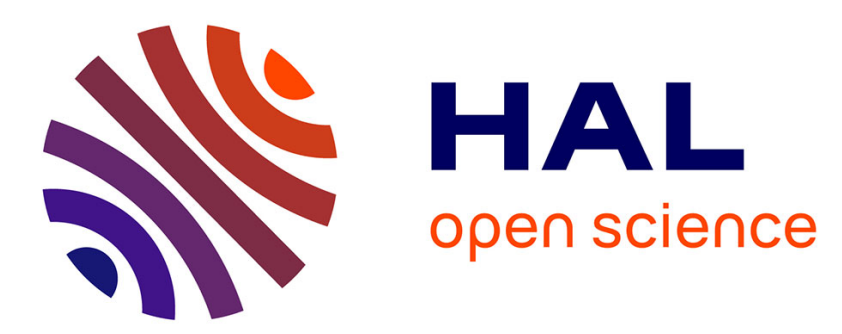

\title{
Considering the attractor structure of chaotic maps for observer-based synchronization problems
}

\author{
Gilles Millérioux, Floriane Anstett, Gérard Bloch
}

\section{To cite this version:}

Gilles Millérioux, Floriane Anstett, Gérard Bloch. Considering the attractor structure of chaotic maps for observer-based synchronization problems. Mathematics and Computers in Simulation, 2005, 68 (1), pp.67-85. 10.1016/j.matcom.2004.10.001 . hal-00111695

\section{HAL Id: hal-00111695 \\ https://hal.science/hal-00111695}

Submitted on 6 Nov 2006

HAL is a multi-disciplinary open access archive for the deposit and dissemination of scientific research documents, whether they are published or not. The documents may come from teaching and research institutions in France or abroad, or from public or private research centers.
L'archive ouverte pluridisciplinaire HAL, est destinée au dépôt et à la diffusion de documents scientifiques de niveau recherche, publiés ou non, émanant des établissements d'enseignement et de recherche français ou étrangers, des laboratoires publics ou privés. 


\title{
Considering the attractor structure of chaotic maps for observer-based synchronization problems
}

\author{
G. Millerioux ${ }^{\text {a } 1}$, F. Anstett ${ }^{\text {a }}$, G. Bloch ${ }^{\text {a }}$ \\ a Centre de Recherche en Automatique de Nancy CRAN (CNRS UMR 7039) \\ ESSTIN, 2, Rue Jean Lamour 54519 Vandoeuvre-Les-Nancy (France)
}

\begin{abstract}
The main purpose of this paper is to state some sufficient conditions for global synchronization of chaotic maps. The synchronization is viewed as a state reconstruction problem which is tackled by polytopic observers. Unlike most standard observers, polytopic observers can account for a special property of chaotic dynamics. Indeed, it is shown that many chaotic maps can be described in a so-called convexified form, involving a time-varying parameter which depends on the chaotic state vector. Such a form makes it possible to incorporate knowledge on the structure of the compact set wherein the parameter lies. This set depends implicitly on the structure of the chaotic attractor. It is proved that the conservatism of the polyquadratic stability conditions for the state reconstruction, stated in a companion paper, can be reduced when the corresponding Linear Matrix Inequalities involve the vertices of the minimal convex hull of this set. Theoretical developments along with special emphasis on computational aspects are provided and illustrated in the context of adaptive synchronization.
\end{abstract}

Key words: polytopic observers, chaos synchronization, minimal convex hull

\section{Introduction}

Chaotic behavior is one of the most complex dynamics that a nonlinear discretetime system (usually named a map) can exhibit. There exist a number of specific properties of chaotic motions as sensitivity to initial conditions, density of periodic orbits in the corresponding attractor, and transitivity [1]. One of the other interesting specificity of chaotic motion is that the corresponding state vector lies in a compact set.

$\overline{1}$ Corresponding author Email: millerioux@esstin.uhp-nancy.fr 
For chaos control, the property of sensitive dependence on initial conditions has been exploited. Indeed, chaos control consists in designing a control law stabilizing the system around one of the unstable periodic orbit embedded in the chaotic attractor. The most well-known method to achieve this control is the OGY method [2]. In contrast with standard control theory, it exploits the specific sensitivity to initial conditions of chaotic systems by designing a local feedback control that makes use of time-dependent perturbations on an accessible parameter of the system.

Historically, chaos synchronization has exhibited an increasing interest in the last decade since the pioneering works of Pecora and Carroll [3][4]. Thereafter, it has entered the control scene and has become a popular open problem from the control theoretic point of view [5]. In [6], an attempt was made to give a general formalism for synchronization in dynamical systems and many special issues devoted to the subject are of particular interest [7][8][9][10]. Formally, achieving synchronization with a so-called drive chaotic system through a unidirectional coupling consists in designing a so-called response system in such a way that, based on some partial information, they exhibit the same behavior. Such an issue has been presented from a control theoretic point of view as a state reconstruction problem and has constituted a milestone for deeper insights. A relevant survey on chaos synchronization dealing with the observer approach can be found in [11]. For recent advanced techniques to achieve synchronization, the reader is referred to [12] for observers with linearizable dynamics, to [13] for observers derived from the concept of absolute stability , to [14] for observers dedicated to systems having a Generalized Hamiltonian Form, or to a plethora of papers dealing with the Extended Kalman Filter based methods. However, at the present, these general state reconstruction approaches don't really take into account the specificity of the chaotic motion, and particularly the fact that the corresponding state vector lies in a compact set, as mentioned previously. Given a chaotic attractor, the state vector is bounded and the bounds can be component wise a priori known. Recently, such a fact has been partially taken into consideration in [16] where the concept of polytopic observers has been introduced. This concept extends some earlier works which were confined to piecewise linear systems [17].

The objective of this paper is to go further in the previous consideration by providing deeper insights on polytopic observers. Considering that a broad class of chaotic maps can admit an affine state description with a possible time-varying dynamical matrix depending on the state vector, the state reconstruction issue of chaotic maps can be formulated as a special observer problem for Linear Parameter Varying (LPV) systems, the time-varying parameter being a function of the state vector. The stability conditions of the state reconstruction error and the computation of the observer gain is derived from the solution of Linear Matrix Inequalities. Here, what pursues and improves the works of [16] is the fact that the polytopic observer design is not only based upon the boundedness of the state vector but incorporates a knowledge of the structure of the chaotic attractor. This is a key point which 
allows us to reduce the conservatism of the stability conditions and, hence, which permits us to enlarge the class of chaotic maps used for synchronization purposes. This paper is self-consistent to a large extent and is an attempt to provide a kind of tutorial on polytopic observers. Its layout is the following. In Section 2, we show how some chaotic maps can admit a convexified form by means of a suitable change of variable. In Section 3, conditions on the global convergence of the state reconstruction error for such systems are recalled. Then, in Section 4, a result allowing the reduction of the conservatism of those conditions is stated by considering the minimal convex hull of the polytope wherein the chaotic time-varying parameter lies. Such a consideration turns out to be of particular interest in the context of adaptive synchronization described in Section 5. Finally, an illustrative example is provided in Section 6 .

\section{Notation and definitions}

$\Omega$ : the attractor wherein the state vector $x_{k}$ of a chaotic dynamical system lies

$\Omega_{\rho}$ : the compact set wherein a time-varying parameter $\rho_{k}$ lies

$\mathcal{D}_{\rho}$ : the polytope wherein the compact set $\Omega_{\rho}$ is embedded

$\mathcal{D}_{\mathcal{A}}$ : the polytope wherein a time-varying matrix $\mathcal{A}$ is embedded

Definition 1. A convex hull of a set of points is the set of all convex combinations of these points.

Definition 2. A convex polytope is the intersection of a finite set of closed halfspaces or equivalently a convex polytope is the convex hull of a finite set of points.

Definition 3. An element of a finite set of points is an extreme point if it is not a convex combination of other points in this set.

Definition 4. A compact set is a bounded and closed set.

\section{Convexifying some chaotic maps}

\subsection{Various classes of chaotic maps}

A generic nonlinear structure that can describe all chaotic systems does not exist. Indeed, many nonlinear systems with a suitable parameter settings can exhibit chaotic motions. Hence, when considering chaos synchronization as a state reconstruction problem, it is difficult to derive a generic observer design method to achieve the synchronization. By now, we often check for some classes of systems for which standard state reconstruction techniques exist independently from the motion, say stationary, periodic or chaotic (see [12] for output injections in the discrete-time case, [14] for systems having Hamilto- 
nian Forms). When a generic observer design is considered, see for instance the Extended Kalman Filter [15], the conditions of global convergence are often difficult to obtain.

Throughout this paper, we are interested in various nonlinear maps but which admit a common affine state description:

$$
\left\{\begin{array}{l}
x_{k+1}=A\left(x_{k}\right) x_{k}+E\left(y_{k}\right) \\
y_{k}=C x_{k}
\end{array}\right.
$$

where $x_{k} \in \mathbb{R}^{n}$ is the state vector, $y_{k} \in \mathbb{R}^{m}$ is the output vector. $A$ and $E$ are, respectively, a $n \times n$ time-varying matrix and a $n$-dimensional vector which depends on the whole state $x_{k}$ or a part of $x_{k} . C \in \mathbb{R}^{m \times n}$ is the output matrix. Many of the usual classes of chaotic maps obey (1). Indeed, set $A\left(x_{k}\right)=A^{*}$ a constant matrix amounts to consider Lur'e systems. Set $A\left(x_{k}\right)=A_{i}$ and $E\left(y_{k}\right)=E_{i}$, some constant matrices assigned, with a one-to-one correspondence, to the regions $R_{i}$ of the state space, visited by $x_{k}$ at the discrete time $k$ and fulfilling $\bigcup_{i=1}^{i=N} R_{i} \subseteq \mathbb{R}^{n}$. It amounts to consider piecewise linear systems. When the dependence on $x_{k}$ of $A$ is polynomial, description (1) corresponds to polynomial chaotic systems like the Logistic map, the Henon map, the Mandelbrot map, ....

An appealing fact is that any discrete trajectory embedded in a chaotic attractor lies in a compact set. It is an invariant set of the phase space and consequently, only a limited part of the characteristic of the nonlinearity is actually visited. In this section, it is shown that for the different classes of usual chaotic maps generically described by (1), it is possible to find an equivalent and unified description of their dynamics, called "convexified form", whenever the state vector lies in the chaotic attractor. Such a generic description will make possible to derive a systematic and tractable observer design with global convergence properties.

The next subsection gives a condition for chaotic maps (1) to obtain an equivalent "convexified" description through a polytopic decomposition.

\subsection{Polytopic decomposition}

We shall say that the nonlinear dynamical system (1) admits an equivalent convexified form if it can be rewritten:

$$
\left\{\begin{array}{l}
x_{k+1}=\mathcal{A}\left(\rho_{k}\right) x_{k}+E\left(y_{k}\right) \\
y_{k}=C x_{k}
\end{array}\right.
$$


with

$$
\mathcal{A}\left(\rho_{k}\right)=\sum_{i=1}^{N} \xi_{k}^{(i)}\left(\rho_{k}\right) \bar{A}_{i}
$$

The vector $\xi_{k}$ belongs to the compact set $\mathcal{S}=\left\{\mu_{k} \in \mathbb{R}^{N}, \mu_{k}=\left(\mu_{k}^{(1)}, \ldots, \mu_{k}^{(N)}\right)^{T}\right.$, $\mu_{k}^{(i)} \geq 0 \forall i$ and $\left.\sum_{i=1}^{N} \mu_{k}^{(i)}=1\right\}$. Owing to the convexity of $\mathcal{S}$, the whole set of matrices $\bar{A}_{i}$ defines a polytope, denoted $\mathcal{D}_{\mathcal{A}}$, with corresponding convex hull denoted $\operatorname{Co} \mathcal{A}\left\{\bar{A}_{1}, \ldots, \bar{A}_{N}\right\}$. The $\bar{A}_{i}$ 's are constant matrices and are named vertices.

It is worth emphasizing that for Lur'e systems or piecewise linear systems, convexifying is quite easy since expressing (1) like (2) is straightforward, the polytopic decomposition being unique [17]. This section aims at stating some conditions under which a polytopic decomposition may exist for chaotic maps (1) with a general dependence (polynomial for instance) on $x_{k}$. This is the issue of Proposition 1. Its proof is constructive in the sense that it provides an explicit expression of the vertices of the polytopic decomposition with respect to the time-varying parameter.

Proposition 1. For (1), when $x_{k}$ lies in a chaotic attractor $\Omega$, if there exists a function $h: \mathbb{R}^{n} \rightarrow \mathbb{R}^{L}$ defined as $\rho_{k}=h\left(x_{k}\right)$ such that :

i) $\mathcal{A}$ defined as $\mathcal{A}\left(\rho_{k}\right)=\mathcal{A}\left(h\left(x_{k}\right)\right)=A\left(x_{k}\right)$ is of class $C^{1}$ with respect to $\rho_{k}$

ii) $\rho_{k}$ is bounded when $x_{k}$ is bounded

then there exist an integer $N$, some $\xi_{k} \in \mathcal{S}$ and some $\bar{A}_{i}$ such that $\mathcal{A}\left(\rho_{k}\right)$ admits the polytopic decomposition (3).

Proof. In the proof, the dependence on $\rho_{k}$ of the $\xi_{k}^{(i)}$,s will be omitted for convenience.

On one hand, assume that there exists a function $h$ such that $\mathcal{A}$, fulfilling $\mathcal{A}\left(\rho_{k}\right)=\mathcal{A}\left(h\left(x_{k}\right)\right)=A\left(x_{k}\right)$, is of class $C^{1}$ with respect to $\rho_{k}=h\left(x_{k}\right)$. Hence, $\mathcal{A}$ can be rewritten in the linear form

$$
\mathcal{A}\left(\rho_{k}\right)=\tilde{A}_{0}+\sum_{j=1}^{L} \rho_{k}^{(j)} \tilde{A}_{j}
$$

$\tilde{A}_{0}$ is the matrix derived from $\mathcal{A}$ by keeping its constant entries while setting to zero its time-varying entries. Let $l$ and $c$ be two indices depending on $j$ and corresponding respectively to the position of $\rho_{k}^{(j)}$ in $\mathcal{A}$. Then, $\tilde{A}_{j}$ is the matrix whose entries are all zero except the one, located at row $l$ and column $c$, which equals unity.

On the other hand, since the system (1) is chaotic, then $x_{k}$ lies in a compact set $\Omega$, and since $h$ ensures $\rho_{k}$ to be bounded when $x_{k}$ is bounded, thus $\rho_{k}$ also 
belongs to a compact set $\Omega_{\rho}$, the image of $\Omega$ under $h$. Hence, there always exist an integer $N$ and some $\xi_{k} \in \mathcal{S}$ such that :

$$
\rho_{k}=\sum_{i=1}^{N} \xi_{k}^{(i)} \bar{\rho}_{i}
$$

meaning that $\Omega_{\rho}$ can be embedded in a polytope $\mathcal{D}_{\rho}$ with convex hull $\operatorname{Co} \rho\left\{\bar{\rho}_{1}, \ldots, \bar{\rho}_{N}\right\}$.

Substituting (5) into (4) yields :

$$
\mathcal{A}\left(\rho_{k}\right)=\tilde{A}_{0}+\sum_{j=1}^{L}\left(\sum_{i=1}^{N} \xi_{k}^{(i)} \bar{\rho}_{i}^{(j)}\right) \tilde{A}_{j}
$$

Since $\sum_{i=1}^{N} \xi_{k}^{(i)}=1$ and $\tilde{A}_{0}$ is constant, $\tilde{A}_{0}=\sum_{i=1}^{N} \xi_{k}^{(i)} \tilde{A}_{0}$ and the new expression reads :

$$
\mathcal{A}\left(\rho_{k}\right)=\sum_{i=1}^{N} \xi_{k}^{(i)}\left(\tilde{A}_{0}+\sum_{j=1}^{L} \bar{\rho}_{i}^{(j)} \tilde{A}_{j}\right)
$$

Finally, identifying (7) with (3) gives the matrices $\bar{A}_{i}$

$$
\bar{A}_{i}=\tilde{A}_{0}+\sum_{j=1}^{L} \bar{\rho}_{i}^{(j)} \tilde{A}_{j}
$$

Remark 1. Equation (3) means that $\mathcal{A}$ lies in a polytope $\mathcal{D}_{\mathcal{A}}$ with convex hull $\operatorname{Co} \mathcal{A}$. It should be noted that the structure of such a polytope depends implicitly on the structure of the chaotic attractor $\Omega$. Indeed, $\mathcal{D}_{\mathcal{A}}$ depends on $\mathcal{D}_{\rho}$, the polytope wherein the time-varying parameter $\rho_{k}$ lies, $\rho_{k}$ being a function of the state vector $x_{k}$ lying in $\Omega$.

The obtained polytopic decomposition ensures the possibility to design in a systematic way a global synchronization by a state reconstruction as it is shown in the next section.

\section{Global synchronization with polytopic observers}

The issue of a state reconstruction for the convexified map (2) can be formulated similarly than for LPV systems. This is discussed below and then, the chaotic specificity is considered. 


\subsection{State reconstruction of convexified chaotic maps}

Under the assumption that the Proposition 1 is fulfilled, the nonlinear dynamical (1) admits an equivalent convexified form (2). Now, assume in addition that $\rho_{k}$ is a quantity available through the output $y_{k}$ and so acts as an on-line time-varying parameter. For the reconstruction of the state $x_{k}$, the following observer can be suggested :

$$
\left\{\begin{array}{l}
\hat{x}_{k+1}=\mathcal{A}\left(\rho_{k}\right) \hat{x}_{k}+E\left(y_{k}\right)+\mathcal{L}\left(\rho_{k}\right)\left(y_{k}-\hat{y}_{k}\right) \\
\hat{y}_{k}=C \hat{x}_{k}
\end{array}\right.
$$

where $\mathcal{L}$ is a time-varying gain, depending on the available time-varying parameter $\rho_{k}$. It it is straightforward to show that the state reconstruction error $\epsilon_{k} \triangleq x_{k}-\hat{x}_{k}$ obtained from (2) and (9) is governed by :

$$
\epsilon_{k+1}=\left(\mathcal{A}\left(\rho_{k}\right)-\mathcal{L}\left(\rho_{k}\right) C\right) \epsilon_{k}
$$

In this way, the dynamics of the state reconstruction is linear in $\epsilon_{k}$ but is time-varying since the matrices depend on the parameter $\rho_{k}$. Thus, (10) can be viewed as a special LPV system whose global stability has to be ensured. We are now looking for sufficient conditions of the global stability of the null solution of (10).

It can be guaranteed by a suitable choice of the gain matrix $\mathcal{L}$ and a key stage is the search for an adequate Lyapunov function. A usual approach is to choose a single quadratic Lyapunov function [18][19]. This approach suffers from conservatism since it does not take into account the parameter variations. In some cases, it can cause the problem to become infeasible, meaning that quadratic stabilization cannot be achieved. A significant improvement can be obtained by considering Parameter Dependent Lyapunov Functions (PDLF) which incorporate the parameter variations. A special parameter dependence is the affine one [20][21] and can be extended to a polynomial one [22]. Unfortunately, affine parameter dependent Lyapunov functions lead to an infinite number of constraints because all the values of the parameters which continuously vary in some prescribed range have to be considered. Thus, one must resort to griding the range of all admissible values in order to obtain a finite set of constraints or to use the multiconvexity concept. Another usual dependence is the polytopic one which allows to overpass the griding and to turn the problem into the resolution of a finite set of constraints by only considering the vertices of the polytope.

Such a discussion motivates the reason why, when possible, the generic class of chaotic systems (1) is rewritten in a polytopic form (Section 2.2) and motivates 
the central choice of $\mathcal{L}$ obeying :

$$
\mathcal{L}\left(\rho_{k}\right)=\sum_{i=1}^{N} \xi_{k}^{(i)}\left(\rho_{k}\right) \bar{L}_{i}
$$

This means that $\mathcal{L}$ is enforced to take values in the polytope $\mathcal{D}_{\mathcal{L}}$ with convex hull $\operatorname{Co} \mathcal{L}\left\{\bar{L}_{1}, \ldots, \bar{L}_{N}\right\}$. $\xi_{k}$ has to coincide with the one involved in the polytopic decomposition (5). This constraint can be always fulfilled since $\xi_{k}$ depends on $\rho_{k}$ which is on-line available. Expressing $\mathcal{L}$ like (11) causes (10) to turn into :

$$
\epsilon_{k+1}=\sum_{i=1}^{N} \xi_{k}^{(i)}\left(\bar{A}_{i}-\bar{L}_{i} C\right) \epsilon_{k}
$$

which represents the dynamics of a system having a polytopic form. And yet, as stressed in the previous discussion, the global stability of the null solution of (12) can be guaranteed by a finite set of convex constraints. This is the purpose of the next theorem which guarantees the global convergence of $\epsilon_{k}$ towards zero through the existence of a special Lyapunov function, called polyquadratic [23]. Besides, this theorem involves a finite set of Linear Matrix Inequalities (LMI) constraints from which the gains $L_{i}$ of (11) are derived.

Theorem 1. System (12) converges globally to zero if there exist some symmetric matrices $P_{i}$, matrices $G_{i}$ and $F_{i}$ such that, $\forall(i, j) \in\{1, \ldots, N\} \times$ $\{1, \ldots, N\}$, the following set of Linear Matrix Inequalities is feasible

$$
\left[\begin{array}{cc}
P_{i} & (\bullet)^{T} \\
G_{i} \bar{A}_{i}-F_{i} C & G_{i}+G_{i}^{T}-P_{j}
\end{array}\right]>0
$$

and $\mathcal{L}\left(\rho_{k}\right)=\sum_{i=1}^{N} \xi_{k}^{(i)} \bar{L}_{i}$ with $\bar{L}_{i}=G_{i}^{-1} F_{i}$

It can be shown that the time-varying gain $\mathcal{L}\left(\rho_{k}\right)=\sum_{i=1}^{N} \xi_{k}^{(i)} \bar{L}_{i}$ with $\bar{L}_{i}=$ $G_{i}^{-1} F_{i}$ ensures the existence of a Lyapunov function $V: \mathbb{R}^{n} \rightarrow \mathbb{R}^{+}$, defined by $V\left(\epsilon_{k}, \xi_{k}\right)=\epsilon_{k}^{T} \mathcal{P}_{k} \epsilon_{k}$ with $\mathcal{P}_{k}=\sum_{i=1}^{N} \xi_{k}^{(i)} P_{i}$ and $\xi_{k} \in \mathcal{S}$, called poly-quadratic Lyapunov function which fulfills :

$$
V\left(\epsilon_{k+1}, \xi_{k+1}\right)-V\left(\epsilon_{k}, \xi_{k}\right)<0 \quad \forall \xi_{k} \in \mathcal{S}, \quad \forall k
$$

Eq. (14) is equivalent to poly-quadratic stability of (12) and is sufficient to global asymptotic stability. The detailed proof is omitted since it is similar to the one given in [17] where pure piecewise linear systems were considered. Nevertheless, the formulation (13), despite of its strict equivalence, differs slightly from the one encountered in [17]. This new formulation is interesting in that (13) no longer needs to manipulate the transpose of the matrices $\bar{A}_{i}$ and $C$ (or equivalently the dual system). 
If we are interested in designing an observer with a constant gain $\bar{L}^{*}$, we can keep on solving the LMIs (13) while replacing $G_{i}$ and $F_{i}$ by some respective constant matrices $G^{*}$ and $F^{*}$. Obviously, the conservatism of the conditions increases but not drastically. Indeed, the extra matrix $G^{*}$ is of first importance in this case because it allows to enforce the gain $\bar{L}^{*}$ to be constant without enforcing the Lyapunov matrices $P_{i}$ to be constant too. The reason is that the gain does not depend explicitly on the Lyapunov matrices since $\bar{L}^{*}=\left(G^{*}\right)^{-1} F^{*}$.

Remark 2. (14) must be fulfilled for all $\xi_{k}$ in $\mathcal{S}$, that is for all $\mathcal{A}\left(\rho_{k}\right) \in \mathcal{D}_{\mathcal{A}}$ or for all $\rho_{k} \in \mathcal{D}_{\rho}$. It means that (14) must be fulfilled not only for all admissible trajectories of $\rho_{k} \in \mathcal{D}_{\rho}$ (corresponding to all admissible trajectories of $x_{k}$ in $\Omega$ with respect to the chaotic motion) but also for any "virtual" trajectories as long as they stay in $\mathcal{D}_{\rho}$. It is the reason why the conditions are only sufficient conditions of synchronization and, in this sense, are conservative.

The following corollary will be useful in next section.

Corollary 1. In order the set of LMI's (13) to be fulfilled, it is necessary that, for any $\rho_{k} \in \mathcal{D}_{\rho}$, the pair $\left(\mathcal{A}\left(\rho_{k}\right), C\right)$ is detectable (i.e. for any given time $k$, there exists a constant gain $\bar{L}^{*}$ such that all the eigenvalues of $\mathcal{A}\left(\rho_{k}\right)-\bar{L}^{*} C$ lies strictly inside the unit circle)

Proof. According to the Remark 2, the feasibility of (13) is equivalent to (14) not only for all admissible but also for all virtual trajectories of $\rho_{k}$ in $\mathcal{D}_{\rho}$. In particular, (14) must hold for the special case $\rho_{k}=\rho^{\star}$ and so $\xi_{k}=\xi^{\star}$, some constant vectors, even though the system is chaotic. This amounts to consider the stability of (12) with the constant dynamical matrix $\mathcal{A}\left(\rho^{\star}\right)-\mathcal{L}\left(\rho^{\star}\right) C$, corresponding to a pure linear system for which the detectability of the pair $\left(\mathcal{A}\left(\rho^{\star}\right), C\right)$ is a necessary condition of stability.

Remark 2 motivates the issue of next section where the problem of the reducing of the conservatism of the sufficient conditions involved in Theorem 1 is addressed. 


\section{Optimal polytopic decomposition}

\subsection{Existence of an optimal polytope}

In [17], it was shown that polyquadratic stability allows us to reduce the conservatism of the stability conditions compared with the usual quadratic stability which corresponds to the special case $G_{i}=P_{i}=P^{*}$ in (13), $P^{*}$ being a constant positive definite matrix. It has been stressed so far that for Lur'e systems or piecewise linear systems, convexifying is immediate since the polytopic decomposition is unique. Thus, for that case, the constraints (13) are also unique. On the other hand, for the general convexified chaotic maps (2), the polytope $\mathcal{D}_{\mathcal{A}}$ with convex hull $\operatorname{Co} \mathcal{A}$ depends on the structure of the chaotic attractor $\Omega$, as mentioned in Remark 1 , and is no longer unique. This subsection aims at showing how, based on the Remark 2, we can obtain some stability conditions (13) the least conservative as possible by computing a socalled optimal polytope.

It has been emphasized in the proof of Proposition 1 that, owing to the specificity of the chaotic motions, the time-varying parameter $\rho_{k}$ in (2) is constrained to a compact set $\Omega_{\rho}$ which can always be embedded in a polytope $\mathcal{D}_{\rho}$.

Proposition 2. For the convexified chaotic map (2), the least conservative conditions (13) ensuring the poly-quadratic stability of (12) are obtained for the matrices $\bar{A}_{i}$, computed through (8) and derived from the $\bar{\rho}_{i}$ corresponding to the minimal convex hull of the polytope $\mathcal{D}_{\rho}$ wherein $\Omega_{\rho}$ is embedded. This polytope is called optimal and is denoted $\mathcal{D}_{\rho}^{*}$.

Proof. All along the proof, we consider an arbitrary polytope $\mathcal{D}_{\rho}$, in $\mathbb{R}^{L}$, wherein $\Omega_{\rho}$ is embedded as depicted on Figure 1 .

On one hand, assume that there exist some particular $\rho_{k} \in \mathcal{D}_{\rho}$ for which (14) is not fulfilled (Figure 1a). Consider any polytope $\mathcal{D}_{\rho}^{\prime}$ which fulfills $\mathcal{D}_{\rho} \subset \mathcal{D}_{\rho}^{\prime}$. Hence, all these $\rho_{k}$ are also included in $\mathcal{D}_{\rho}^{\prime}$ and (14) doesn't still hold by replacing $\mathcal{D}_{\rho}$ into $\mathcal{D}_{\rho}^{\prime}$. Conversely, consider any polytope $\mathcal{D}_{\rho}^{\prime}$ which fulfills $\Omega_{\rho} \subseteq \mathcal{D}_{\rho}^{\prime} \subset \mathcal{D}_{\rho}$. As a consequence, it can be expected that $\mathcal{D}_{\rho}^{\prime}$ no longer contains the previous particular $\rho_{k}$ (Figure 1b). As a result, (14) is likely to be fulfilled for $\rho_{k}$ in $\mathcal{D}_{\rho}^{\prime}$.

On the other hand, assume that there aren't any $\rho_{k} \in \mathcal{D}_{\rho}$ for which (14) is not fulfilled. Consider any polytope $\mathcal{D}_{\rho}^{\prime}$ which fulfills $\mathcal{D}_{\rho} \subset \mathcal{D}_{\rho}^{\prime}$. Hence, it may exist some $\rho_{k}$ in $\mathcal{D}_{\rho}^{\prime}$ such that (14) no longer holds (Figure 1c). Conversely, considering any polytope $\mathcal{D}_{\rho}^{\prime}$ which fulfills $\Omega_{\rho} \subseteq \mathcal{D}_{\rho}^{\prime} \subset \mathcal{D}_{\rho}$, it cannot exist some 
$\rho_{k} \in \mathcal{D}_{\rho}^{\prime}$ preventing (14) to be fulfilled (Figure 1d).

To conclude, the optimal polytope is the one corresponding to the minimal convex hull wherein $\Omega_{\rho}$ is embedded.

a)
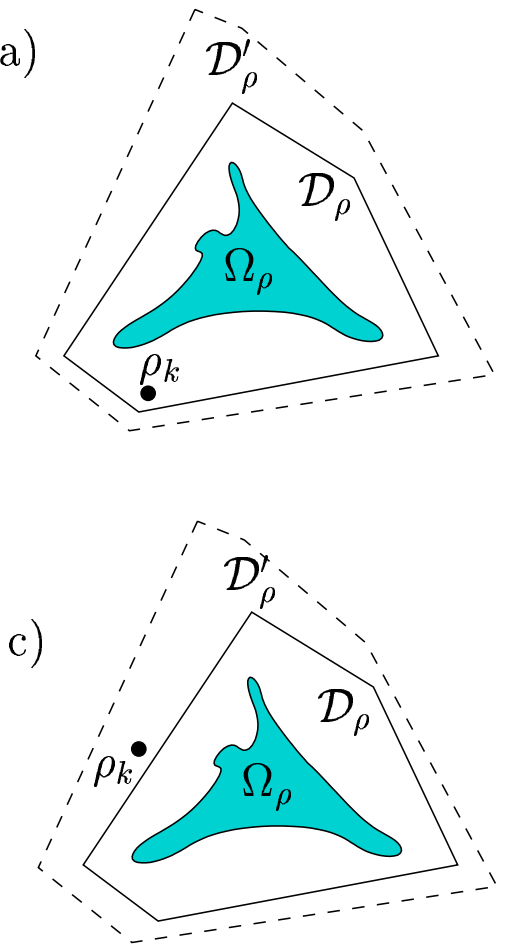

b)
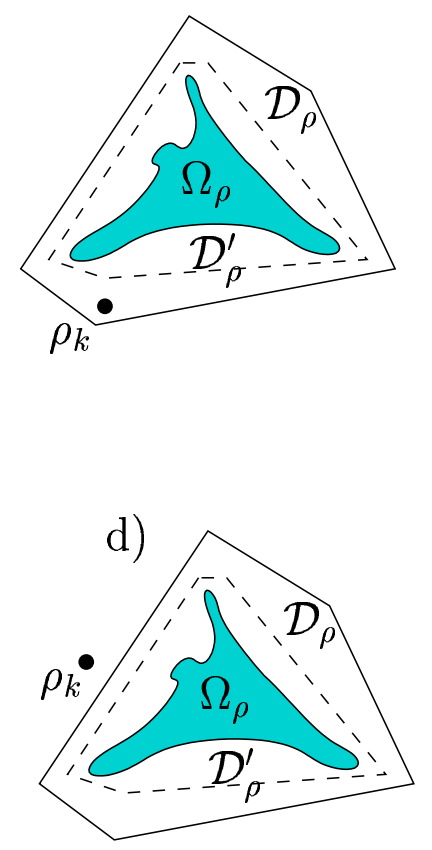

Fig. 1. Attractor $\Omega_{\rho}$ and embedding polytopes $\mathcal{D}_{\rho}$ and $\mathcal{D}_{\rho}^{\prime}$ in $\mathbb{R}^{L}$.

Remark 3. This proposition highlights the fact that the feasibility of LMI's is not related to the number of constraints. Indeed, all considerations have been made irrespective of the number of vertices.

In the sequel, a method for the computation of the minimal polytope, or equivalently the minimal convex hull, is provided.

\subsection{Computation of the minimal convex hull}

The computation of the minimal convex hull $\mathcal{D}_{\rho}^{*}$ can be performed by standard methods and before proceeding any further, they are briefly reminded.

The enumeration of all vertices of a convex polytope is a central issue in mathematical programming and computational geometry. The computation of the convex hull in dimension 2 has been studied extensively and several efficient algorithms are available. The most popular is the Graham Scan [24]. It is based on the consideration that the angle between two consecutive faces (formed by three consecutive vertices) of the convex hull is lower than $\pi$. As the the number of points $N_{p}$ in the set increases, the complexity is $\sigma\left(N_{p} \log N_{p}\right)$. 
This algorithm has a main drawback in that it cannot be extended to dimensions greater than 2. Another efficient algorithm called Quick hull is based on the "divide and conquer" approach. It has been introduced in slightly different forms by [25] and [26]. This algorithm uses the following property with a great efficiency: given a triangle of three points of the original set, the points inside this triangle do not belong to the convex hull. Hence, they can be discarded. Like the Graham Scan, this algorithm is also very efficient (complexity close to $\sigma\left(N_{p} \log N_{p}\right)$ ). Moreover it can be easily extended to any dimension (see [27] for the dimension 3). However, as the number of operations grows with the dimension, the complexity increases $\left(\sigma\left(N_{p}^{2}\right)\right.$ for dimension 3$)$.

Some algorithms use simple projections iteratively to find the extreme points [28]. Other algorithms use linear programming formulations of the convex hull problem [29], but that becomes equivalent to solving an optimization problem.

For the problem considered in this paper, the computation of the optimal polytope $\mathcal{D}_{\rho}^{*}$ is done once from a set of points generated by simulating (1) over a significant time interval. We recall that the resulting vertices $\bar{A}_{i}$ are introduced in the constraints (13) from which the gains $\bar{L}_{i}$ are derived. All those operations can be carried out off-line. On the other hand, the computation of the observer gain (11) requires an on-line computation of the $\xi_{k}^{(i)}$ 's which is now discussed.

\subsection{On-line decomposition}

The computation of the observer gain (11) must involve the same coefficients $\xi_{k}^{(i)}$ than the ones involved in the polytopic decomposition (5) of the timevarying parameter $\rho_{k}$. Computing $\xi_{k}$ involved in (5) requires solving at each discrete-time $k$ the constrained problem :

$$
\min _{\xi_{k}}\left\|E_{k}-F G_{k}\right\| \quad \text { subject to } \xi_{k}^{(i)} \geq 0,1 \leq i \leq N
$$

with

$$
E_{k}=\left[\begin{array}{l}
\rho_{k}^{(1)} \\
\vdots \\
\rho_{k}^{(j)} \\
\vdots \\
\rho_{k}^{(L)} \\
1
\end{array}\right], \quad F=\left[\begin{array}{ccccc}
\bar{\rho}_{1}^{(1)} & \ldots & \bar{\rho}_{i}^{(1)} & \ldots & \bar{\rho}_{N}^{(1)} \\
\vdots & \vdots & \vdots & \vdots & \vdots \\
\bar{\rho}_{1}^{(j)} & \ldots & \bar{\rho}_{i}^{(j)} & \ldots & \bar{\rho}_{N}^{(j)} \\
\vdots & \vdots & \vdots & \vdots & \vdots \\
\bar{\rho}_{1}^{(L)} & \ldots & \bar{\rho}_{i}^{(L)} & \ldots & \bar{\rho}_{N}^{(L)} \\
1 & \ldots & 1 & \ldots & 1
\end{array}\right], \quad G_{k}=\left[\begin{array}{l}
\xi_{k}^{(1)} \\
\vdots \\
\xi_{k}^{(j)} \\
\vdots \\
\xi_{k}^{(N)}
\end{array}\right]
$$


$F$ is a time-invariant $(L+1) \times N$ matrix and results from the previous off-line computation of $\mathcal{D}_{\rho}^{*}$. $E_{k}$ is a known $(L+1)$-dimensional vector since $\rho_{k}$ is on-line available. It is reminded that the $\xi_{k}$ belongs to the convex set $\mathcal{S}$. The entries related to the last row of the matrices correspond to the equality constraint $\sum_{i=1}^{N} \xi_{k}^{(i)}=1$. The positivity constraint is included in (15).

Such a problem is a standard constrained optimization problem for which usual routines exist but a specific alternative method can be suggested for our special purpose. The procedure is explained for the case $L=2$ but can be directly extended to higher dimension. The key point is that, unlike the general case, the strict equality $E_{k}=F G_{k}$ is ensured at each discrete-time $k$. Indeed, since $\rho_{k}$ lies in the chaotic attractor $\Omega_{\rho}$ which is entirely embedded in the polytope $\mathcal{D}_{\rho}^{*}$, the existence of the $\xi_{k}$ 's fulfilling the positivity constraints is ensured. Actually, there exists an infinity of solutions but an optimal solution from a computational complexity point of view can be obtained. Considering that at each time $k, \rho_{k}$ is strictly included inside the minimal convex polytope $\mathcal{D}_{\rho}^{*}$, it is also located inside a triangle shaped by 3 vertices of this polytope denoted $\bar{\rho}_{p}, \bar{\rho}_{q}, \bar{\rho}_{r}$. Hence, $\rho_{k}$ can be written as a convex combination of this 3 vertices whose coefficients are derived from the reduced unconstrained equality :

$$
\underline{E}_{k}=\underline{F G}_{k}
$$

with

$$
\underline{E}_{k}=\left[\begin{array}{c}
\rho_{k}^{(1)} \\
\rho_{k}^{(2)} \\
1
\end{array}\right], \quad \underline{F}=\left[\begin{array}{ccc}
\bar{\rho}_{p}^{(1)} & \bar{\rho}_{q}^{(1)} & \bar{\rho}_{r}^{(1)} \\
\bar{\rho}_{p}^{(2)} & \bar{\rho}_{q}^{(2)} & \bar{\rho}_{r}^{(2)} \\
1 & 1 & 1
\end{array}\right], \underline{G}_{k}=\left[\begin{array}{c}
\xi_{k}^{(p)} \\
\xi_{k}^{(q)} \\
\xi_{k}^{(r)}
\end{array}\right]
$$

The remaining $(N-3)$ coefficients $\xi_{k}^{(i)}$ are set to zero. The constant matrix $F$ is square and of full rank such that its inverse always exists. It is obtained from a prior search for an admissible triangle which can be carried out by the Delaunay method [30] for instance.

The proposed technique for the on-line decomposition, through (16), can be extended to dimensions $L$ greater than 2 in a straightforward manner but the increase of complexity of the Delaunay method with the dimension may cause a loss of interest.

We conclude that computing the minimal convex hull $\mathcal{D}_{\rho}^{*}$ reduces the conservatism of global synchronization conditions. Such a result turns out to be of particular interest in the context of adaptive synchronization which is now presented. 


\section{Adaptive chaos synchronization}

Adaptive chaos synchronization is a relevant issue which is widely investigated in the literature [31] [32]. Since it is not the main purpose of the paper, omitting the corresponding bibliography is a deliberate choice. It consists in retrieving not only the whole state vector of a chaotic system but also some unknown or uncertain parameters. This situation is encountered when attempting to synchronize two chaotic systems with neither identical initial conditions nor identical parameters. Parameter mismatch is a realistic and problematic situation in engineering applications, and in some communication schemes, the mismatch is intentional. This is typically the case when a parameter is modulated according to a rule which encodes the information to be transmitted as in Chaos Shift Keying [33].

Many works usually resort to the Extended Kalman Filter (EKF) to handle the problem as a joint state and parameter estimation one. In [34], a new adaptive synchronization scheme was suggested within the framework of polytopic observers. This approach is presented as an alternative to the well-known Extended Kalman Filter. In this section, an in-depth study of the conditions under which a global adaptive synchronization can be achieved is carried out as an illustration of the results stated so far.

We focus on the set of chaotic systems of class $C^{1}$ with some unknown parameter $\theta \in \mathbb{R}^{p}$. Thus, those systems can be rewritten as :

$$
\left\{\begin{array}{l}
x_{k+1}=A\left(x_{k}\right) x_{k}+f\left(x_{k}\right) \theta+E\left(y_{k}\right) \\
y_{k}=C x_{k}
\end{array}\right.
$$

with $f$ a function from $\mathbb{R}^{n}$ to $\mathbb{R}^{n \times p}$ of $x_{k}$. Such a class has been introduced in [34] and enlarges the class usually considered for the joint state and parameter estimation problem where the dynamical matrix $A$ is constant. By introducing an extended state vector denoted $\bar{x}_{k}=\left[x_{k}^{T} \theta_{k}^{T}\right]^{T}$, taking into account that $\theta$ is constant, the system can be rewritten :

$$
\left\{\begin{array}{l}
\bar{x}_{k+1}=\bar{A}\left(x_{k}\right) \bar{x}_{k}+\bar{E}\left(y_{k}\right) \\
y_{k}=\bar{C} \bar{x}_{k}
\end{array}\right.
$$

with

$$
\bar{A}\left(x_{k}\right)=\left[\begin{array}{cc}
A\left(x_{k}\right) & f\left(x_{k}\right) \\
\mathbf{0} & \mathbf{1}
\end{array}\right], \quad \bar{E}\left(y_{k}\right)=\left[\begin{array}{c}
E\left(y_{k}\right) \\
\mathbf{0}
\end{array}\right], \quad \bar{C}=\left[\begin{array}{ll}
C & \mathbf{0}
\end{array}\right]
$$

$\mathbf{1}$ and $\mathbf{0}$ are respectively the identity and the null matrices of proper dimension. Applying Proposition 1 to the augmented system (18) and assuming that it is 
fulfilled, (18) can be rewritten :

$$
\left\{\begin{array}{l}
\bar{x}_{k+1}=\overline{\mathcal{A}}\left(\rho_{k}\right) \bar{x}_{k}+\bar{E}\left(y_{k}\right) \\
y_{k}=\bar{C} \bar{x}_{k}
\end{array}\right.
$$

with

$$
\overline{\mathcal{A}}\left(\rho_{k}\right)=\left[\begin{array}{cc}
\mathcal{A}\left(\rho_{k}\right) & \mathcal{F}\left(\rho_{k}\right) \\
\mathbf{0} & \mathbf{1}
\end{array}\right]=\sum_{i=1}^{N} \xi_{k}^{(i)}\left(\rho_{k}\right) \bar{A}_{i}
$$

where $\mathcal{A}\left(\rho_{k}\right)=\mathcal{A}\left(h\left(x_{k}\right)\right)=A\left(x_{k}\right)$ and $\mathcal{F}\left(\rho_{k}\right)=\mathcal{F}\left(h\left(x_{k}\right)\right)=f\left(x_{k}\right)$. It is reminded that $\rho_{k}$ is a time-varying parameter lying in a compact set $\Omega_{\rho}$ which can always be embedded in a polytope $\mathcal{D}_{\rho}$.

Thus, the following polytopic observer can be used :

$$
\left\{\begin{array}{l}
\hat{\bar{x}}_{k+1}=\overline{\mathcal{A}}\left(\rho_{k}\right) \hat{\bar{x}}_{k}+\bar{E}\left(y_{k}\right)+\overline{\mathcal{L}}\left(\rho_{k}\right)\left(y_{k}-\hat{y}_{k}\right) \\
\hat{y}_{k}=\bar{C} \hat{\bar{x}}_{k}
\end{array}\right.
$$

with $\overline{\mathcal{L}}\left(\rho_{k}\right)=\sum_{i=1}^{N} \xi_{k}^{(i)}\left(\rho_{k}\right) \bar{L}_{i}$. The state reconstruction error equation is governed by :

$$
\epsilon_{k+1}=\left(\overline{\mathcal{A}}\left(\rho_{k}\right)-\overline{\mathcal{L}}\left(\rho_{k}\right) \bar{C}\right) \epsilon_{k}
$$

Taking into account the polytopic decomposition of the matrices $\overline{\mathcal{A}}$ and $\overline{\mathcal{L}}$, (22) turns into :

$$
\epsilon_{k+1}=\sum_{i=1}^{N} \xi_{k}^{(i)}\left(\rho_{k}\right)\left(\bar{A}_{i}-\bar{L}_{i} \bar{C}\right) \epsilon_{k}
$$

The global convergence of (23) is ensured by applying Theorem 1 with the new matrices $\bar{A}_{i}, \bar{C}$ and the corresponding gains $\bar{L}_{i}$.

Proposition 3. In order that the system (23) converges poly-quadratically toward zero, it is necessary that $\mathcal{F}\left(\rho_{k}\right)$ never vanishes whatever $\rho_{k}$ belonging to $\mathcal{D}_{\rho}$.

Proof. $\overline{\mathcal{A}}\left(\rho_{k}\right)$ and $\overline{\mathcal{L}}\left(\rho_{k}\right)$ are rewritten in the following partitioned matrices:

$$
\overline{\mathcal{A}}\left(\rho_{k}\right)=\left[\begin{array}{cc}
A_{11} & A_{12} \\
\mathbf{0} & \mathbf{1}
\end{array}\right], \quad \overline{\mathcal{L}}\left(\rho_{k}\right)=\left[\begin{array}{l}
L_{1} \\
L_{2}
\end{array}\right]
$$

with $A_{11}=\mathcal{A}\left(\rho_{k}\right), A_{12}=\mathcal{F}\left(\rho_{k}\right), L_{1}$ and $L_{2}$ of compatible sizes. Reminding that $\bar{C}=\left[\begin{array}{ll}C & \mathbf{0}\end{array}\right]$ yields :

$$
\overline{\mathcal{A}}\left(\rho_{k}\right)-\overline{\mathcal{L}}\left(\rho_{k}\right) \bar{C}=\left[\begin{array}{cc}
A_{11}-L_{1} C & A_{12} \\
-L_{2} C & \mathbf{1}
\end{array}\right]
$$


If there exist some $\rho_{k} \in \mathcal{D}_{\rho}$ such that $A_{12}=\mathcal{F}\left(\rho_{k}\right)$ vanishes, the characteristic polynomial of $\overline{\mathcal{A}}\left(\rho_{k}\right)-\overline{\mathcal{L}}\left(\rho_{k}\right) \bar{C}$, namely $\psi(\lambda)=\operatorname{det}\left(\overline{\mathcal{A}}\left(\rho_{k}\right)-\overline{\mathcal{L}}\left(\rho_{k}\right) \bar{C}-\lambda \mathbf{1}\right)$ is :

$$
\psi(\lambda)=(1-\lambda)^{p} \cdot \operatorname{det}\left(A_{11}-L_{1} C-\lambda \mathbf{1}\right)
$$

with $\lambda \in \mathbb{C}$. Clearly, $\lambda=1$ will be a solution of $\psi(\lambda)=0$. Such an eigenvalue prevents the detectability condition of Corollary 1 and so the polyquadratic stability of (23) to be fulfilled.

Actually, according to the Proposition 2, the optimal polytope is the one corresponding to the minimal convex hull wherein $\Omega_{\rho}$ is embedded to prevent $\mathcal{F}\left(\rho_{k}\right)$ from vanishing.

\section{Illustrative example}

Consider the following nonlinear map derived from [35].

$$
\left\{\begin{array}{l}
x_{k+1}^{(1)}=\cos \varphi x_{k}^{(1)}-\sin \varphi x_{k}^{(2)} \\
x_{k+1}^{(2)}=\sin \varphi x_{k}^{(1)}+(\cos \varphi-0.3 \alpha) x_{k}^{(2)}+2 \alpha \cdot\left(x_{k}^{(2)}\right)^{2}+4 \theta \alpha \cdot\left(\left(x_{k}^{(2)}\right)^{3}+b\right) \\
y_{k}=x_{k}^{(2)}
\end{array}\right.
$$

This map (24) is of the form (17) where:

$$
\begin{aligned}
& A\left(x_{k}\right)=\left[\begin{array}{cc}
\cos \varphi & -\sin \varphi \\
\sin \varphi & \cos \varphi-0.3 \alpha+2 \alpha x_{k}^{(2)}
\end{array}\right], \\
& f\left(x_{k}\right)=4 \alpha\left(\left(x_{k}^{(2)}\right)^{3}+b\right), \\
& E\left(y_{k}\right)=0, \\
& C=\left[\begin{array}{ll}
0 & 1
\end{array}\right]
\end{aligned}
$$

For $\varphi=3.03, \theta=1$, when the pair $(\alpha, b)$ belongs to the set of admissible values depicted in Figure 2, (24) exhibits a chaotic motion. The corresponding chaotic attractor $\Omega$ for $\alpha=2.7$ and $b=0.005$ is shown in Figure 3 .

We want to achieve a chaos synchronization based on an observer approach despite of the fact that $\theta$ is assumed to be only known with a bounded uncertainty $\Delta=0.05$. As a consequence, we must resort to an adaptive synchronization to retrieve simultaneously the unmeasurable state $x_{k}^{(1)}$ and the unknown parameter $\theta$. 


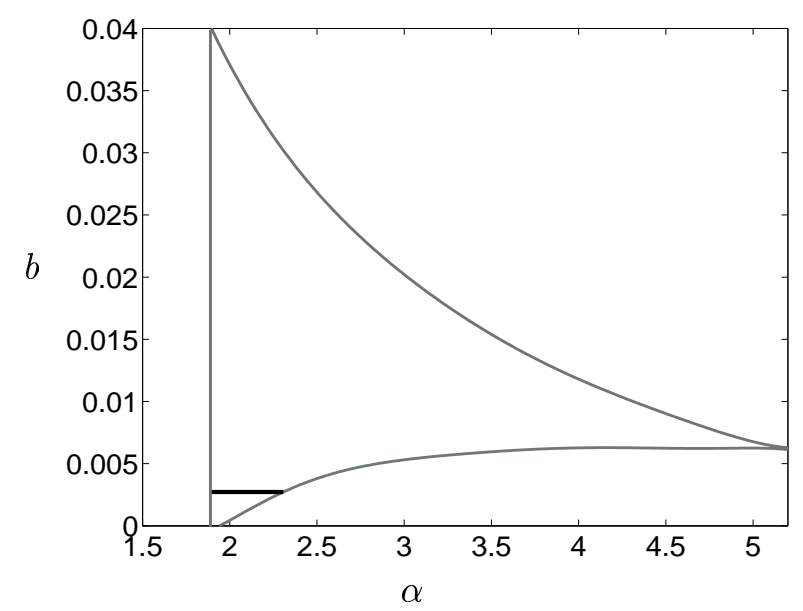

Fig. 2. Inside the gray contour : the set of admissible pairs $(\alpha, b)$ corresponding to a chaotic motion for $(24)$.

[Black straight line : the set of pairs corresponding to a loss of detectability $\left(\rho_{k}^{(2)}=0\right)$, see the discussion of case 1.]

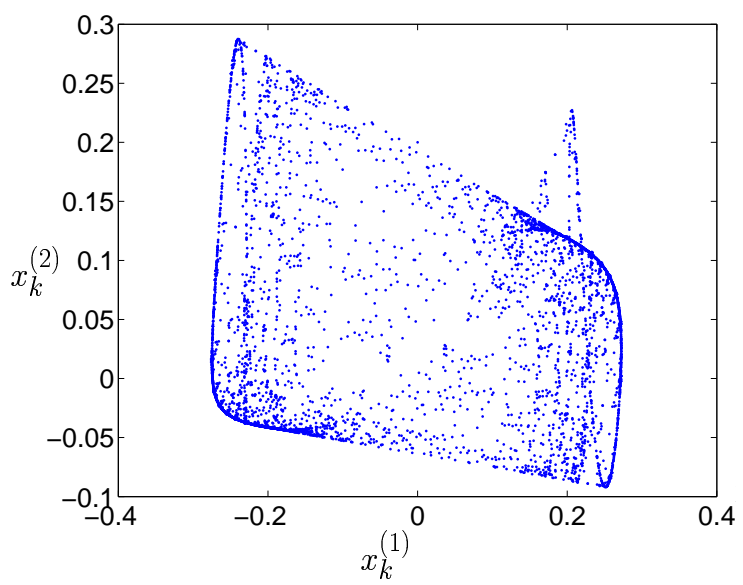

Fig. 3. Chaotic attractor $\Omega$ in the $\left(x_{k}^{(1)}, x_{k}^{(2)}\right)$ plane for $\alpha=2.7$ and $b=0.005$.

Two distinct choices of embedding polytope $\mathcal{D}_{\rho}$ are addressed.

\section{Case 1: Optimal polytope}

\section{Step 1}

Taking the function $h$ defined as $\rho_{k}^{(1)}=h_{1}\left(x_{k}\right)=\cos \varphi-0.3 \alpha+2 \alpha x_{k}^{(2)}$ and $\rho_{k}^{(2)}=$ $h_{2}\left(x_{k}\right)=4 \alpha\left(\left(x_{k}^{(2)}\right)^{3}+b\right),(24)$ can be rewritten in the convexified augmented 
state space form (18) with $\bar{x}_{k}=\left[x_{k}^{T} \theta_{k}^{T}\right]^{T}$ and the matrices :

$$
\overline{\mathcal{A}}\left(\rho_{k}\right)=\left[\begin{array}{ccc}
\cos \varphi & -\sin \varphi & 0 \\
\sin \varphi & \rho_{k}^{(1)} & \rho_{k}^{(2)} \\
0 & 0 & 1
\end{array}\right], \quad \bar{E}\left(y_{k}\right)=\left[\begin{array}{l}
0 \\
0 \\
0
\end{array}\right], \quad \bar{C}=\left[\begin{array}{lll}
0 & 1 & 0
\end{array}\right]
$$

Here, $\mathcal{F}\left(\rho_{k}\right)=\left[\begin{array}{ll}0 & \rho_{k}^{(2)}\end{array}\right]^{T}$. It is easy to see that $h$ fulfills the assumptions $\left.i\right)$ and $i$ ) of Proposition 1. The function $h$ also satisfy the additional assumption made in subsection 3.1, that is $\rho_{k}$ being a quantity available through the output $y_{k}$.

\section{Step 2}

Since $x_{k}$ is chaotic, $x_{k}$ lies in a chaotic attractor $\Omega$ and thus $\rho_{k}$ evolves in the mapped compact set $\Omega_{\rho}$, that is the image of $\Omega$ under $h$ depicted in Figure 4 . Let note that the structure of $\Omega_{\rho}$ is smooth. It accounts for the fact that the function $h$ is polynomial. To obtain $\Omega_{\rho}$, taking into account the uncertainty $\Delta$ on $\theta$, eq. (24) has been simulated over a significant time interval for different values of $\theta$ "gridded" within the range $[1-\Delta, 1+\Delta]$. The $\rho_{k}$ has been collected and the Graham Scan [24] has been used to compute the vertices of the optimal polytope $\mathcal{D}_{\rho}^{*}$. The algorithm gives $N=97$ vertices (see the corresponding polytope on the Figure 4 ). Then, the $\bar{A}_{i}$ 's are computed in a straightforward

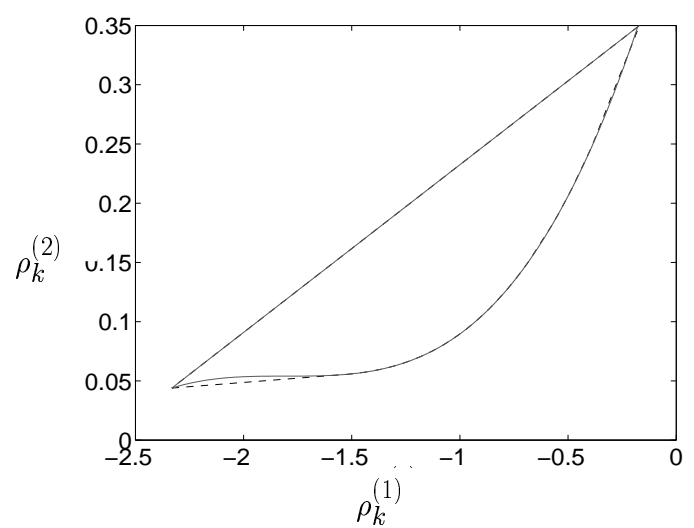

Fig. 4. Mapped compact set $\Omega_{\rho}$ (solid line) and its corresponding embedding convex polytope $\mathcal{D}_{\rho}^{*}$ in the $\left(\rho_{k}^{(1)}, \rho_{k}^{(2)}\right)$ plane (dashed line).

way according to (8) with

$$
\tilde{A}_{0}=\left[\begin{array}{ccc}
\cos \varphi & -\sin \varphi & 0 \\
\sin \varphi & 0 & 0 \\
0 & 0 & 1
\end{array}\right], \quad \tilde{A}_{1}^{l c}=\left[\begin{array}{lll}
0 & 0 & 0 \\
0 & 1 & 0 \\
0 & 0 & 0
\end{array}\right], \quad \tilde{A}_{2}^{l c}=\left[\begin{array}{lll}
0 & 0 & 0 \\
0 & 0 & 1 \\
0 & 0 & 0
\end{array}\right]
$$

\section{Step 3}


The LMI's (13) with the previously computed $\bar{A}_{i}$ are solved. They turn out to be feasible and the gains $\bar{L}_{i}=G_{i}^{-1} F_{i}$ are derived from their solution.

\section{Discussion}

Let check whether the necessary condition of Proposition 3 is actually fulfilled. If so, it means that $\mathcal{F}\left(\rho_{k}\right)$ (or equivalently $\rho_{k}^{(2)}$ ) never vanishes whatever $\rho_{k}$ belonging to $\mathcal{D}_{\rho}^{*}$. According to Figure $4, \mathcal{D}_{\rho}^{*}$ doesn't cross the straight line $\rho_{k}^{(2)}=0$. As a matter of fact, it is true for any pairs $(\alpha, b)$ of the map (24) located above this straight line $b=0.0027$, reported on the parameter plane of Figure 3.

The determination of the time-varying observer gain (11) requires an on-line computation with a simple matrix inversion as detailed in subsection 4.3. Figure 5 and Figure 6 show the convergence to zero of the synchronization error between the state $x_{k}^{(1)}$ and the estimated state $\hat{x}_{k}^{(1)}$ and between the state $x_{k}^{(2)}$ and the estimated state $\hat{x}_{k}^{(2)}$. Finally, Figure 7 shows the convergence of the estimated parameter $\hat{\theta}_{k}$ toward the true parameter $\theta=1.01$. The simulations are consistent with the theoretical results since the recovering of both the unmeasured state and the uncertain parameter is successfully achieved. Let recall that since the convergence is global, it is ensured whatever the initial conditions of the observer are.

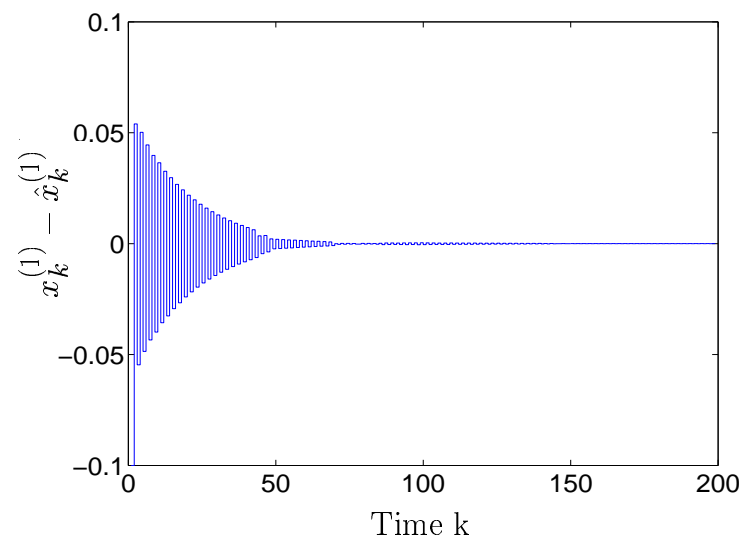

Fig. 5. Synchronization error for $x_{k}^{(1)}-\hat{x}_{k}^{(1)}$.

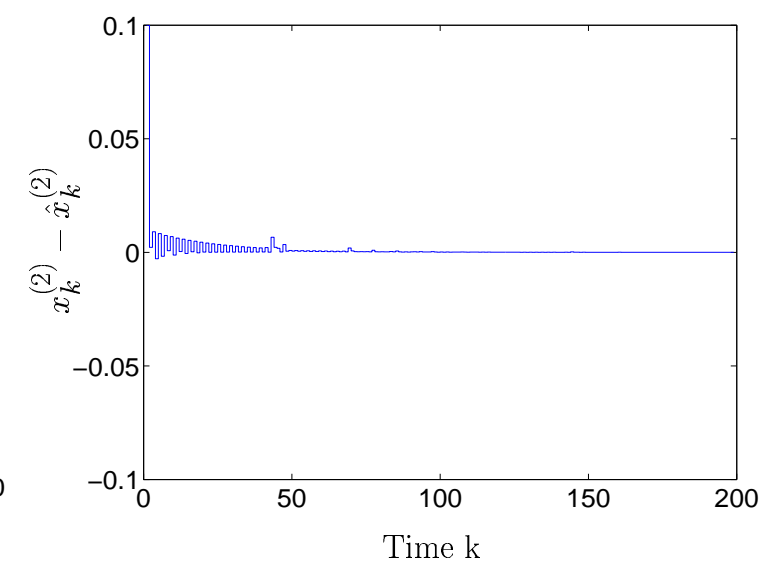

Fig. 6. Synchronization error for $x_{k}^{(2)}-\hat{x}_{k}^{(2)}$.

\section{Case 2: Polytope with low number of vertices}

It should be interesting to wonder whether embedding $\Omega_{\rho}$ in a rectangle of 4 vertices would be wiser than a polytope having 97 vertices. The choice of such a polytope is rather similar of the choice (proposed in [16]) considering the 


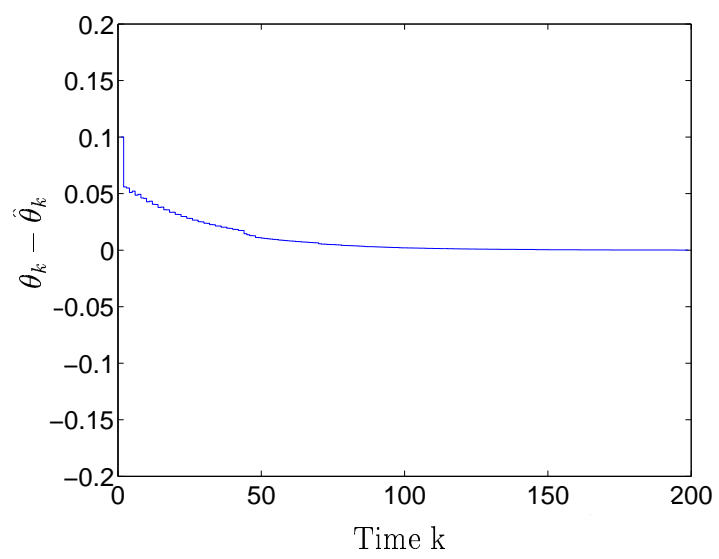

Fig. 7. Synchronization error for $\theta_{k}-\hat{\theta}_{k}$.

extreme values of the components of the time-varying vector. Steps 1 and 2 have been performed again with the 4-vertices polytope depicted in Figure 8.

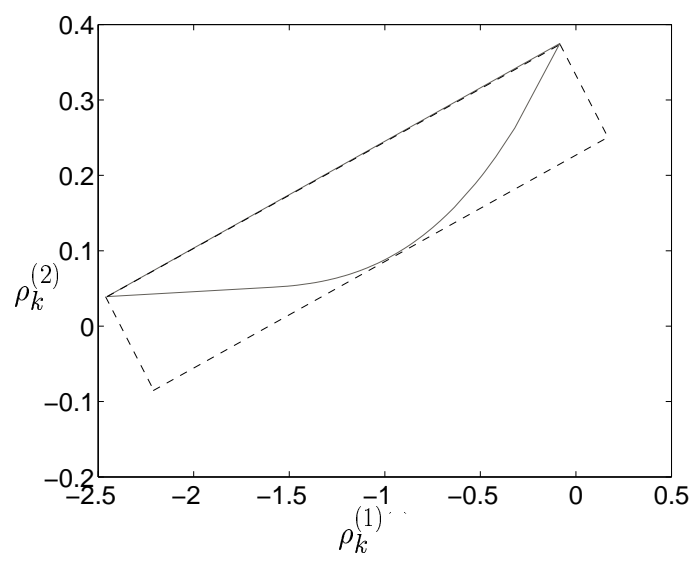

Fig. 8. Minimal rectangle (dashed line) and minimal convex polytope (solid line).

The LMI (13) with the new vertices $\bar{A}_{i}$ have no solutions in that case and illustrates the consistency of the Proposition 2.

\section{Discussion}

We have to check whether $\rho_{k}^{(2)}$ never vanishes when $\rho_{k} \in \mathcal{D}_{\rho}$. According to Figure 8 , for such an embedding, the polytope $\mathcal{D}_{\rho}$ crosses the straight line $\rho_{k}^{(2)}=0$. It highlights the reason why the optimal polytope in the sense of the minimal convex hull incorporating the mapped chaotic attractor $\Omega_{\rho}$, and not in the sense of the minimal number of vertices, have to be chosen. It corroborates the main theoretical result of the paper concerning the search for a minimal polytope in order to get the least conservative conditions. 


\section{Conclusion}

The problem of observer-based chaos synchronization was addressed. The use of polytopic observers allow us to incorporate one of the specificity of chaotic motion, the fact that the corresponding state vector lies in a compact set. Theoretical results along with special emphasis on computational aspects have been provided. In this paper, it has been shown that the conservatism of the polyquadratic stability conditions can be reduced when the corresponding Linear Matrix Inequalities involve the vertices of the minimal convex hull of this set.

As a result, to reduce the conservatism of the conditions, we have now two means at our disposal. The first one consists of the use of polyquadratic stability instead of quadratic stability (a result presented in [17]). From a computational point of view, the former is more demanding since it involves more LMI's than the later but their resolution are made once and off-line. The second one consists of the computation of the minimal convex hull instead of convex hull with minimal number of vertices (the main result of this paper). The number of resulting vertices are often larger than a non minimal one. It causes the number of operations required for the on-line observer gain computation to increase. However, when the computational cost must be taken into consideration, such a drawback can be circumvented by enforcing a constant gain for the observer without drastically increasing the conservatism.

A remaining theoretical matter is to what extent it may exist a change of variable allowing a general chaotic map to be described in a polytopic way involving a dynamical matrix with an on-line time-varying parameter. From a computational point of view, checking for a suboptimal polytope with less vertices could be interesting. The method could be inspired from the ones encountered in set membership problems for instance. Those issues will deserve a more extended treatment and will be the objective of future works.

Acknowledgments. The authors wish to thank J-R Roche of the "Institut de Mathématiques Elie Cartan" of Nancy (IECN) in France to bring to their attention the possible use of Delaunay method.

\section{References}

[1] R. L. Devaney. An Introduction to Chaotic Dynamical Systems. AddisonWesley, Redwood City, CA, 1989.

[2] E. Ott, C. Grebogi, and J. A. Yorke. Controlling chaos. Phys. Rev. Lett., 64(11):1196-1199, 1990. 
[3] L. M. Pecora and T. L. Carroll. Synchronization in chaotic systems. Phys. Rev. Lett., 64:821-824, 1990.

[4] L. M. Pecora and T. L. Carroll. Driving systems with chaotic signals. Phys. Rev. A, 44(8):2374-2383, August 1991.

[5] V. D. Blondel, E. D. Sontag, M. Vidyasagar, and J. C. Willems. Open Problems in Mathematical Systems and Control Theory. Communication and Control Engineering. Springer Verlag, 1999.

[6] I. I. Blekhman, A. .L. Fradkov, H. Nijmeijer, and A. Y. Pogromsky. On selfsynchronization and controlled synchronization. Systems and Control Letters, 31(5):299-305, 1997.

[7] H. Nijmeijer, editor. Special Issue. Control of chaos and synchronization. Syst. Control Letters, 31(5):259-322, 1997.

[8] M. P. Kennedy, M. J. Ogorzalek, editors. Special Issue. Chaos synchronization and control : theory and applications. IEEE Trans. Circuits. Syst. I: Fundamental Theo. Appl, 40:853-1039, 1997.

[9] M. P. Kennedy, M. J. Ogorzalek, editors. Special Issue. Control and synchronization of chaos. International Journal of Bifurcation and Chaos, 10(4), 2000 .

[10] I.I. Blekhman E. Mosekilde, A.L. Fradkov, editor. Special Issue on Chaos Synchronization and Control, volume 58. Elsevier, 2002.

[11] H. Nijmeijer and I. M. Y. Mareels. An observer looks at synchronization. IEEE Trans. Circuits. Syst. I: Fundamental Theo. Appl, 44:882-890, October 1997.

[12] H. J. C. Huijberts, T. Lilge, and H. Nijmeijer. Nonlinear discrete-time synchronization via extended observers. International Journal of Bifurcation and Chaos, 11(7):1997-2006, 2001.

[13] A. Pogromsky and H. Nijmeijer. Observer-based robust synchronization of dynamical systems. International Journal of Bifurcation and Chaos, 8(11):2243-2254, 1998.

[14] H. Sira Ramirez. and C. Cruz Hernandez. Synchronization of chaotic systems :a generalized Hamiltonian approach. International Journal of Bifurcation and Chaos, 11(5):1381-1395, 2001.

[15] C. Cruz and H. Nijmeijer. Synchronization through filtering. International Journal of Bifurcation and Chaos, 110(4):763-775, 2000.

[16] G. Millerioux and J. Daafouz. Polytopic observer for global synchronization of systems with output measurable nonlinearities. International Journal of Bifurcation and Chaos, 13(3):703-712, March 2003.

[17] J. Daafouz and G. Millerioux. Poly-quadratic stability and global chaos synchronization of discrete time hybrid systems. Special Issue of Mathematics and Computers in Simulation, 58:295-307, March 2002. 
[18] G. Becker, A. Packard, D. Philbrick, and G. Balas. Control of parametricallydependent linear systems : a single quadratic Lyapunov approach. In Proc. of American Control Conference, San Fransisco, June 1993.

[19] L. El Ghaoui and S-I. Niculescu, editors. Advances in Linear Matrix Inequality Methods in Control. SIAM's Advances in Design and Control. 2000.

[20] P. Gahinet, P. Apkarian, and M. Chilali. Parameter-dependent lyapunov functions for real parametric uncertainty. IEEE Trans. on Automatic Control, 41(3):436-442, 1996.

[21] E. Feron, P. Apkarian, and P. Gahinet. Analysis and synthesis of robust control systems via parameter-dependent Lyapunov functions. IEEE Trans. on Automatic Control, 41:1041-1046, July 1996.

[22] P-A. Bliman. Nonconservative LMI approach to robust stability for systems with uncertain scalar parameters. In Proc. of 41st IEEE Control and Decision Conference, Las Vegas, USA, December 2002.

[23] J. Daafouz and J. Bernussou. Parameter dependent Lyapunov functions for discrete time systems with time varying parametric uncertainties. Systems and Control Letters, 43:355-359, 2001.

[24] R. L. Graham. An efficient algorithm for determining the convex hull of a finite planar set. Information Processing Letters, 2(1):132-133, 1973.

[25] W. F. Eddy. ACM Trans. Math. Soft, 3(398), 1977.

[26] F. P. Preparata and M. I. Shamos. Computational Geometry. Springer-Verlag, October 1985.

[27] D. C. S. Allison and M. T. Noga. Computing the three-dimensional convex hull. Computer Physics Communications, 103(1):74-82, June 1997.

[28] S. Chatterjee and S. Chatterjee. A note on finding extreme points in multivariate space. Computational statistics and data analysis, 10:87-92, 1990.

[29] P. M. Pardalos, Y. Li, and W. W. Hager. Linear programming approaches to the convex hull problem in $\mathcal{R}^{m}$. Computers Mathematics and Applications, 29(7):23-29, 1995.

[30] C. Du. An algorithm for automatic delaunay triangulation of arbitrary planar domains. Advances in Engineering Software, 27:21-26, 1996.

[31] C. W. Wu, T. Yang and L. O. Chua. On adaptive synchronization and control of nonlinear dynamical systems. International Journal of Bifurcation and Chaos, 6(3):455-471, 1996.

[32] A. L. Fradkov and A. Y. Markov. Adaptive synchronization of chaotic systems based on speed-gradient method and passification. IEEE Trans. Circuits Syst. I: Fundamental Theo. Appl., 44(10):905-912, 1997. 
[33] H. Dedieu, M. P. Kennedy and M. Hasler. Chaos shift keying: modulation and demodulation of a chaotic carrier using self-synchronizing Chua's circuits. IEEE Trans. Circuits Syst. II: Analog and Digital Signal Processing., 40(10):634-642, 1993.

[34] F. Anstett, G. Millerioux, and G. Bloch. Global adaptive synchronization based upon polytopic observers. In Proc. of IEEE International symposium on circuit and systems, ISCAS'04, Vancouver, Canada, May 2004.

[35] I. Gumowski and C. Mira. Dynamique chaotique. Transformations ponctuelles. Transitions Ordre-Désdordre. Cépadues Edition, 1980. 